\title{
A Methodology for Systematic Volumetric Analysis of Perioperative Cranial Imaging in Neurosurgical Patients
}

\author{
Kofi-Buaku Atsina, MDadi'; Richard Gorniak, MD²; Ashwini D. Sharan, MD²; \\ Chengyuan $\mathrm{Wu}, \mathrm{MD}, \mathrm{MSBmEc}^{1}$ \\ ${ }^{1}$ Department of Radiology, Thomas Jefferson University, Philadelphia, PA \\ 2Department of Neurological Surgery, Thomas Jefferson University, Philadelphia, PA
}

\section{KEY WORDS}

Image segmentation, image registration, image analysis, workflow, computer analysis

\begin{abstract}
\section{Background}

Although objective assessment of perioperative imaging provides a rigorous evaluation method of neurosurgical techniques in epilepsy, its use remains far from mainstream. Open surgery remains the gold standard for treatment of mesial temporal lobe epilepsy (MTLE); however, stereotactic laser ablation is a promising minimally invasive alternative. Nevertheless, the variables that may affect seizure outcome in stereotactic laser amygdalohippocampectomy (SLAH) remain unclear. While an objective endpoint such as ablated mesial temporal volumes may be significant, a standard methodology for calculating such volumes has yet to be proposed.
\end{abstract}

\section{Objectives}

To formulate and test a methodology, which can aid in critical evaluation of laser trajectories, and ablation cavities in seizure patients.

\begin{abstract}
Methods
We performed a retrospective study involving 16 patients undergoing SLAH our institution's approved IRB protocol. Preoperative MRIs were processed and segmented. Postoperative MRIs were co-registered to preoperative MRIs. Laser trajectories and ablation cavities were segmented from this co-registered image. Segmented trajectories, and cavities were superimposed upon the initial MRI. The percentage of each structure affected was calculated, using a voxel by voxel comparison.
\end{abstract}

\section{Results}

We were successfully able to determine ablation volumes and critically evaluate laser placement.

\section{Conclusion}

This semi-automated methodology showcases a systematic workflow that objectively evaluates perioperative imaging in neurosurgical patients.

\section{BACKGROUND}

In cranial neurosurgical procedures, postoperative imaging is utilized widely as a method of monitoring and evaluating treatment techniques. Postoperative MR imaging provides the surgeon with feedback that not only informs further management of a patient's illness, but also objectively evaluates the surgical technique used. For cranial neoplasms, abscesses, and vascular lesions, neurosurgeons qualitatively determine the extent of resection and presence of residual lesion based on visual inspection of postoperative MR imaging. With advances in MR technology and computer analysis software, objective assessment of postoperative cranial imaging can be incorporated into the clinical workflow. In this study, we utilize image data sets from epilepsy patients treated at our institution to showcase a systematic methodology by which postoperative imaging can be more objectively assessed.

In epilepsy surgery, open resection constitutes the mainstay of therapy for drug resistant mesial temporal lobe epilepsy (MTLE). Standard anterior temporal lobectomy (ATL) and selective amygdalohippocampectomy (SAH) have been the gold standards of therapy for patients with MTLE, as they have been shown to provide seizure-free outcomes in 60 to $80 \%$ of patients ${ }^{1-3}$. Over recent years, stereotactic radiofrequency ablation (SFRA) 4-10 and stereotactic laser ablation (SLA) 3,11 have been employed as minimally invasive alternatives for the treatment of MTLE, with seizure-freedom rates approaching those for open surgery.

As alluded to above, the critical evaluation of perioperative imaging is paramount as it enables means by which factors affecting post-surgical seizure outcomes are assessed, regardless of the surgical intervention. In 1989, Awad et al, described a 20-compartment model of the temporal lobe in postoperative MRIs as an objective means of assessing how extent of resection correlates with seizure outcomes in patients with temporal lobe epilepsy. ${ }^{12}$ With this very model, Nayel et al., were able to conclude that, the extent of resection correlates positively and is thus, an important determinant in seizure outcomes in patient with baso-mesial 
temporal lobe epilepsy. ${ }^{13}$ Since that time, several investigators have attempted to corroborate the above findings similarly employing MR imaging analysis, but with varied results. ${ }^{14-21}$ The reason for this discrepancy is multi-factorial, not the least of which is that perioperative MR volumetric techniques are varied amongst investigators and thus, thwarts objective comparison amongst studies.

Evaluation of newer surgical techniques such as SRFA and SLA with imaging is not without the aforementioned challenge. In recent years, the emergence of sophisticated computer-assisted MR volumetric analysis has significantly augmented our ability to better define and understand anatomic boundaries of cortical and subcortical structures, and has provided improved evaluation of perioperative imaging. While experience with stereotactic laser amygdalohippocampectomy $(\mathrm{SLAH})$ to date has suggested that seizure outcome may not be directly associated with total ablation volume, ${ }^{3}$ further analysis has suggested that an ablation encompassing the majority of both the amygdala and hippocampus is associated with a greater chance of seizure freedom. ${ }^{10,22}$ While these findings fall in line with the volumetric data from open surgery $13,14,16,17,23$ other temporal lobe structures have yet to be involved in ablation cavity analysis of SLAH. As such, the ideal target and extent of ablation for SLAH has yet to be determined.

Clearly defining anatomical structures from preoperative imaging provides a template for comparison with processed postoperative imaging, enabling further analysis. In the case of SLAH, it enables us to not only demonstrate precise laser location, but also to demonstrate anatomic structures relative to target structures that are otherwise obscured by the effects of the ablation on postablation imaging. This process ultimately allows us to more objectively understand and characterize the variables involved in SLAH. To this end, we formulated and tested a methodology that can be used to critically evaluate laser placement and ablation volumes in SLAH for MTLE.

\section{METHODS}

\section{Subjects}

We performed a retrospective cohort study involving 16 patients who underwent SLAH for MTLE at the Comprehensive Epilepsy Center at Thomas Jefferson University Hospital between 2011 and 2014, under a Thomas Jefferson approved IRB protocol. All patients included in the study were adults, who had lesional temporal lobe epilepsy that had been treated with SLAH [Table 1]. All patients that had extra-temporal epilepsy, laser ablation to non-mesial temporal lobe structures or had inadequate imaging were excluded from the study.

\section{MR ACQUISITION}

All patients underwent both a preoperative $1 \mathrm{~mm}$ slice volumetric non-contrast $\mathrm{T} 1$ weighted MRI, and a postoperative $1 \mathrm{~mm}$ slice volumetric contrast-enhanced T1 weighted MRI using a standardized protocol. All MRI volumetric images were acquired on a Phillips Achieva 3.0T scanner (Phillips Medical System, Andover, MA, USA). Preoperative images were acquired from a sagittal 3D T1 for preoperative functional MR testing and reconstructed as axial images. Technical parameters were as follows: Repetition Time (TR): 6.4 - 6.8 ms; Echo time (TE): 3.1 - 3.3 ms, FOV: $25.6 \mathrm{~cm}$, Flip: 8 degrees; Echo train length (ETL): 240; Matrix size: 256 x 240; Thickness: $1 \mathrm{~mm}$.

\section{Table 1. Patient Characteristics}

\begin{tabular}{|c|c|c|c|c|}
\hline Patient \# & Age & Sex & Case & MR Finding \\
\hline 1 & 56 & F & LSLAH & L MTS \\
\hline 2 & 49 & M & LSLAH & L MTS \\
\hline 3 & 41 & F & LSLAH & L MTS \\
\hline 4 & 42 & F & LSLAH & L MTS \\
\hline 5 & 25 & M & LSLAH & L MTS \\
\hline 6 & 66 & F & LSLAH & L MTS \\
\hline 7 & 54 & F & R SLAH & R MTS \\
\hline 8 & 59 & M & LSLAH & L MTS \\
\hline 9 & 48 & F & R SLAH & R MTS \\
\hline 10 & 19 & M & R SLAH & R MTS \\
\hline 11 & 52 & F & LSLAH & L MTS \\
\hline 12 & 34 & F & LSLAH & L MTS \\
\hline 13 & 52 & F & R SLAH & R MTS \\
\hline 14 & 58 & M & LSLAH & Early L MTS \\
\hline 15 & 29 & M & LSLAH & L MTS \\
\hline 16 & 24 & F & LSLAH & L MTS \\
\hline
\end{tabular}

Postoperative MR images used were obtained at the end of the ablative procedure and contrast was given because the margin of ablation showed enhancement. Technical parameters as follows: Repetition Time (TR): 6.4 - 8.0 ms; Echo time (TE): 2.8 - 3.9 ms, FOV: 25 - 27 cm, Flip: 7 - 8 degrees; Echo train length (ETL): 240; Matrix size: varied; Thickness: $1 \mathrm{~mm}$; Contrast: gadobenate dimeglumine or gadobutrol. 


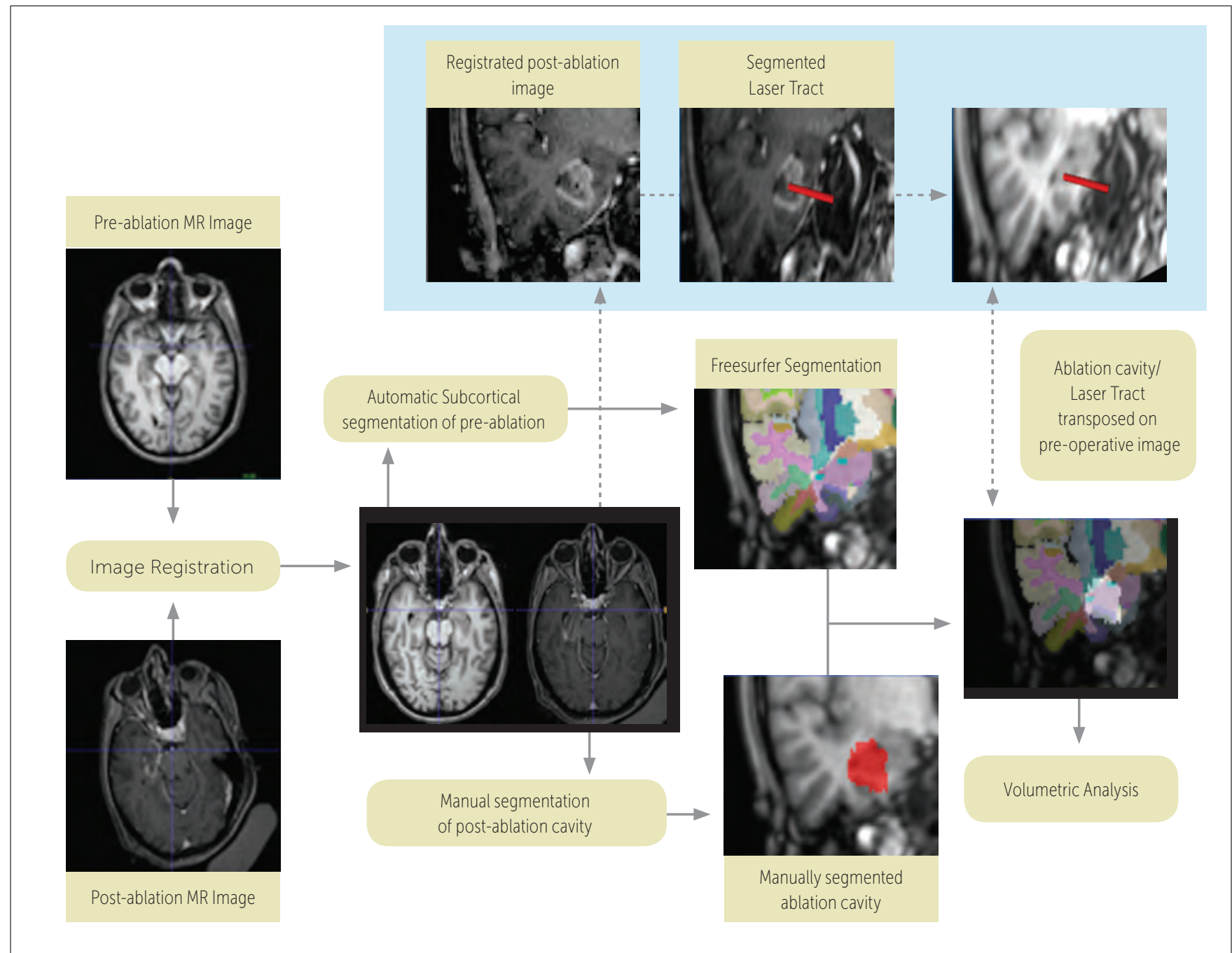

\section{Figure 1}

Workflow of image co-registration, segmentation, laser tract determination, and volumetric analysis od pre- and post- ablation MR images of patients with mesial temporal lobe epilepsy. Adpated from Wu et al. 2015

\section{WORKFLOW}

Pre- and postoperative MR image co-registration, segmentation, as well as volumetric analysis of ablation cavities constitute our workflow as described in Figure 1.

\section{Preoperative Image Processing and Segmentation}

Preoperative non-contrast MR images underwent automatic segmentation with standard anatomic definitions using FreeSurfer, (Martinos Center for Biomedical Imaging, Harvard-MIT, Boston, MA USA; freesurfer.net), ${ }^{24-36}$ which is documented and freely available to download online. As described by Fischl et al., 2002, whole brain segmentation with emphasis on subcortical structures is performed by assigning a neuroanatomical label to each voxel based on probabilistic information automatically estimated from a large training set. Thirty-seven labels are generated, of which eighteen are assigned to subcortical structures and cerebrospinal fluid. ${ }^{37,38}$ Each segmented image was inspected for accuracy before further use. If necessary, corrective steps were taken as described in the FreeSurfer documentation.
Postoperative Image Co-registration

Postoperative MRIs were co-registered to the preoperative images using the "General Registration [BRAINS]" module in 3D Slicer (Surgical Planning Lab, Brigham and Women's Hospital - MIT Artificial Intelligence Laboratory, MA, USA; http:// www.slicer.org). In order to do this, preoperative images were used as reference images. Postoperative T1 weighted MR images were co-registered, whereby their image sets are geometrically transformed by aligning points in the reference image to corresponding points in the postoperative image. 
Using the Insight toolkit (ITK) library, 3D Slicer enables spatial normalization by employing hierarchies of rigid and deformable spatial transformations. ${ }^{39}$ The "Same Subject Brain MRI collection" in the Slicer registration library provided examples with registration parameters that were appropriate for our studies, with a few modifications. Because the shape of the brain does not change much with head movement, we assumed that a rigid registration method would suffice. As such, we estimated a set of parameters that describe the rigid body transformation matrix. Each registration output was reviewed by the senior author (CW) and considered successful if postoperative images were aligned with preoperative images in regions outside of the ablation.

\section{Manual Segmentation of Postoperative image set}

Laser trajectories and ablation cavities were manually segmented from this co-registered image using ITK-SNAP (Penn Image Computing and Science Laboratory, University of Pennsylvania, PA, USA, and the Scientific Computing and Imaging Institute, University of Utah, UT, USA; http://www.itksnap.org). ${ }^{40}$ Manual segmentation was achieved in standard fashion, by tracing boundaries of the ablation cavity on each millimeter slice. Also leveraging the Insight Toolkit (ITK) library, ITK-SNAP's strength lies in image segmentation, and as such was our choice image analysis tool for our studies. Using its overlay modality, the reference images were placed along side the postablation images, thus segmentations could be viewed on both sets of images [Figure 2a,b \& 2c,d]. Because ITK-SNAP allows visualization in three orthogonal planes simultaneously, segmentations were performed in all three panels to ensure precise three-dimensional reconstructions viewable in a fourth pane ${ }^{40,41}$ [Figure 2e; sagittal views not shown].

\section{Volumetric Analysis and Laser Trajectory Determination}

Segmented ablation cavities on postoperative images were superimposed upon their corresponding preoperative MR images to determine the volume of ablation of each anatomical structure [Figure 1]. The volume and percentage
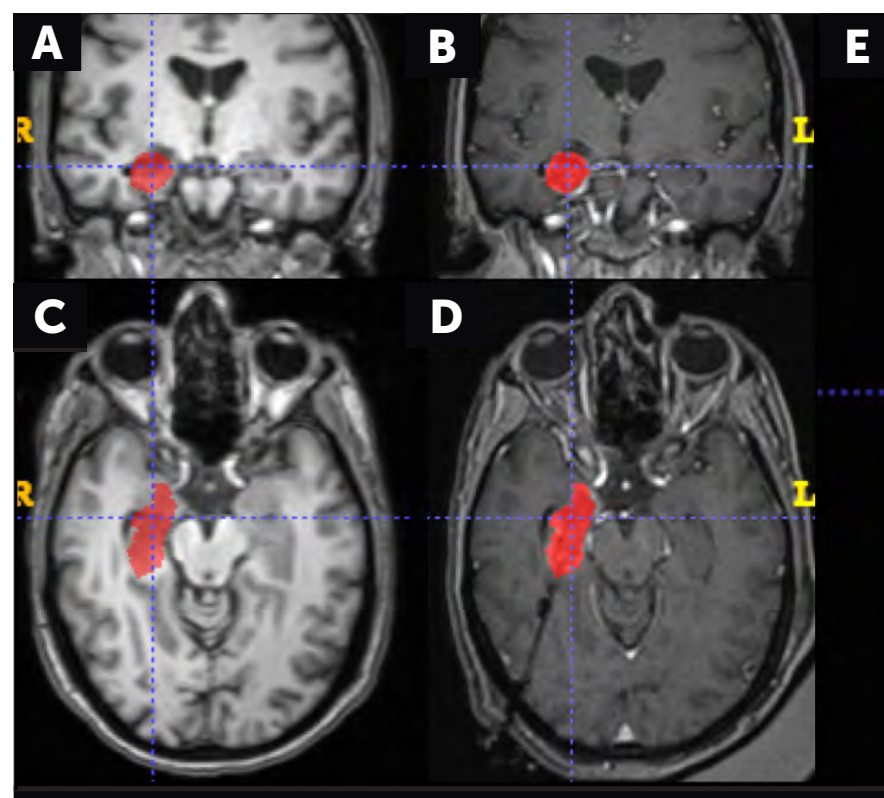

\section{$\mathbf{E}$}

Figure 2

A, B Coronal view of segmented pre- and post- ablation; C, D Axial view of segemented

pre- and post- ablaition; E 3D Volumetric resonstruction of ablation cavity saggittal views are not shown

of each structure affected was calculated by measuring the degree of overlap between the anatomical segmentation completed by Freesurfer and the segmentation of the ablation cavity, using a Matlab-derived script that performed a voxel by voxel comparison of corresponding image sets.

Since the position of the laser relative to anatomic structures cannot be determined once the ablation cavity is present, segmented laser trajectories were similarly superimposed on corresponding preoperative MR images to create a resulting image set. This was in turn used to perform measurements of the laser catheter in relation to relevant anatomic features, enabling us to objectively determine laser position and trajectory as depicted by Wu et al. ${ }^{22}$ [Figure 1]

\section{RESULTS}

We were successfully able to determine volumes of the ablation cavities using this methodology. These volumes were consistent and were verified with careful visual inspection. At each step of the process, outputs from each module of the algorithm were thoroughly examined for errors, which if detected were corrected ultimately producing an objective volumetric assessment. We were additionally able to critically evaluate laser placement by analyzing segmented laser trajectories with preliminary results suggesting the importance of accurate laser placement - with a target in the superior hippocampal head to maximize the length of hippocampus that can be cannulated as shown by Wu et al. ${ }^{22}$

\section{DISCUSSION}

In the present study, we developed a methodology for perioperative imaging analysis and outlined a systematic workflow algorithm that enables objective evaluation of seizure outcomes for ablative neurosurgical techniques employed for epilepsy 
treatment. Specifically, this methodology enables testing of variables that affect seizure outcomes after SLAH including optimal placement of the laser ablation probe, and volumetric estimations of each mesial temporal lobe structure in the region of ablation (i.e. amygdala, hippocampus, and parahippocampal gyrus). It is modular in approach, and relies heavily on computerized three-dimensional volumetric analyses and reconstructions of cranial MR imaging utilizing a variety of automated software.

\section{A Modular Approach}

Despite the fact that we streamlined this methodology utilizing specific image processing and volumetric analysis software, these components are not intrinsic to this algorithm, highlighting its modularity [Figure 3]. As such, any image registration and segmentation software could have been employed. For instance, 3D Slicer and $\mathrm{SPM}^{8}$ in our experience produced similarly excellent product images after co-registration. However, we tended to gravitate towards 3D Slicer because of accessibility, ease of use, and better image display capabilities.

In a similar fashion, we employed Freesurfer for whole brain segmentation because of its wide usage for cortical parcellation, its ease of access, ease of use and familiarity with this manuscript's authors. Our own experience falls in line with prior data by other investigators that Freesurfer sufficiently approximates manually segmented volumes. 37,42-44 Additionally, comparison of automated segmentation methods of subcortical temporal lobe structures with regard to manual segmentation have demonstrated that Freesurfer is more accurate, and shows better volume overlap (Dice coefficient), and higher correlation than other widely used methods such as FSL/ FIRST ${ }^{42,43,45}$ and IBASPM. ${ }^{44,46}$

There nonetheless, remains no consensus as to which automated segmentation methods are superior, however several may be adequate for volumetric analysis. In a study by Akhondi-Asl et al., automated segmentation methods, such as Localinfo and HAMMER supersede Freesurfer in overlap volume and demonstrate relatively higher concordance to manual segmentation methods ${ }^{47}$. Results from another study demonstrated that Freesurfer had poor performance in hippocampal volumetry compared to other automated segmentation methods such as ANIMAL-multi and SACHA ${ }^{48}$.

Given the current evolution in the field of automated segmentation, a modular approach to this methodology allows alternate automated segmentation methods including HAMMER, Localinfo, ANIMAL-multi, SACHA, FS+LDDMM or $A B S S, 38,48,49$ to be used in a similar fashion.

\section{Potential Sources of Error}

All comparisons were done using preand postoperative image sets obtained

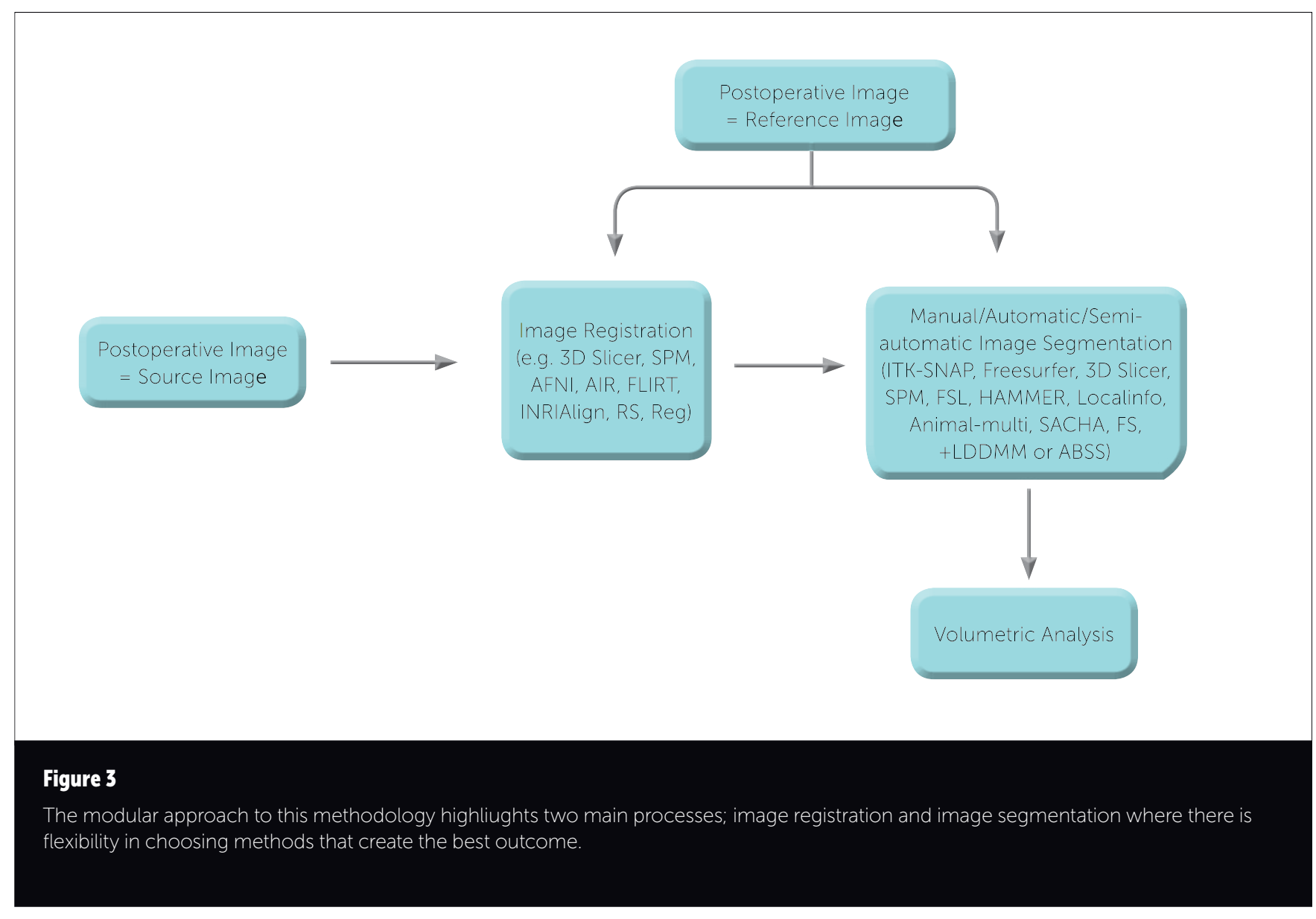


from the same patient. By using patient images as their own controls, we eliminated factors such as gender, body habitus ${ }^{50}$ and duration of epilepsy ${ }^{51,52}$, that may influence amygdala and hippocampal dimensions. Despite this, there is variability introduced at some steps in our workflow, whose overall effect need consideration.

\section{Image Acquisition}

It is well known that the type of MR scanner and MR acquisition technique can significantly affect image registration and segmentation by impacting image resolution, partial volume effects, signalto-noise ratio, intensity inhomogeneities and artifact 53. As such, high-resolution MR images obtained using the same predetermined image acquisition protocols (see methods) was used in pre- and postoperative image analyses, thus minimizing variability amongst registered image sets and reducing the degree of error in segmented image sets. Even with these constraints, Morey et al., albeit based on preliminary studies, suggest the persistence of differences in segmentation outcomes when acquisition of image sets is separated temporally. ${ }^{42}$

MRI is typically able to capture agerelated changes in normal brain. Until about age 50 - 60, global cortical atrophy rate in normal adults remains very gradual ${ }^{54-58}$. In one systematic review of 56 longitudinal MRI studies in healthy participants, cortical atrophy rates were negligible between age 18 and 35 , approximately $0.2 \%$ with a steady rise to $0.5 \%$ between age $35-60$, and $>0.5$ $\%$ after age $60 .{ }^{58}$ Besides normal aging, global cortical atrophy rates have been shown to be higher in chronic refractory epilepsy patients. ${ }^{59-61}$ In our cohort, all pre- and post-ablation images were obtained within a short time (arbitrarily defined as $<6$ months) difference, (range: 6 days -4 months) except in one case (patient \# 14: 2 years). We postulated that shorter time difference between acquisition of pre- and post- treatment images, helped to minimize variability that can be introduced by large changes in brain morphology, shape or size due to aging, refractory epilepsy and other age-related disease.

\section{Manual versus Automated Segmentation}

Although manual segmentation remains the gold standard, how manually obtained volumes compare with true volumes remains unknown, and as such widely debated. Manual segmentation results are affected by anatomical protocols, tracer experience and fatigue, image acquisition protocols and image quality ${ }^{42,62,63}$. Furthermore, it seems reasonable to assume that any two expertly trained technicians with similar experience will end up with some inconsistencies in segmentation based on inherent delineator differences. ${ }^{50}$ While Freesurfer automated segmentation, circumvents some of these issues, comparative studies show that it consistently overestimates volumetric parameters of subcortical and tissue segmentation for both normal and disease states ${ }^{43-46,48,64-66}$ limiting the utility of absolute volumes derived for these structures and introducing error in further downstream analysis. These over-estimations represent a "systematic error" consistently expressed within the Freesurfer segmentation pipeline 44,46 and thus are of little significance in the interpretation of our data. One advantage of the modular approach is that it allows current image processing methods to be replaced by new and better methods that further optimize this workflow algorithm enabling efficiency, and maintaining reproducibility, but without compromising quality.

\section{Changes in Intracranial Architecture}

In our cohort, changes in intracranial architecture were largely insignificant, because the minimally invasive nature of this laser technology ensures minimal egress of cerebrospinal fluid, and consequently results in no significant brain shift. Additionally, because postoperative images were obtained immediately after laser treatment (last MR scan before removing probe, see Figure 2D), there was no perceivable brain shift that could arise from post-surgical brain edema at such an early phase.

\section{Applications and Limitations}

Quantitative analytic technologies have developed hand in hand with advances in imaging technology, as the need for objective and precise interpretation of clinical imaging has become more apparent. In 2008, the National Cancer Institute started to develop the Quantitative Imaging Network with the prime purpose of advancing quantitative imaging, as well as predicting and monitoring treatment response. Computerized analysis has, and continues to aid in their efforts as it allows for reproducibility and efficiency, while still allowing for personalized medicine. ${ }^{67}$ But the advantages of computerized imaging analysis extend far beyond cancer evaluation and treatment, to aiding in our understanding of many different pathological conditions with imaging correlates, not the least of which is brain pathology. In fact, in recent years computerized $M R$ and $C T$ volumetric analysis employing a variety of image analysis software has been used to investigate a gamut of neurological conditions ranging from Alzheimer's, ${ }^{68-70}$ Schizophrenia, ${ }^{38,71}$ Huntington's disease, Tourette's syndrome, ${ }^{38}$ Brain tumor, ${ }^{72-74}$ HIV-related CNS disease ${ }^{44}$ and Epilepsy. 47-49,75-79

The methodology described above streamlines image analysis, in a manner that to our knowledge, has not been previously described. Although our dataset specifically concerned patients who underwent SLAH for MTLE, the methodology can be applied to other clinical situations. For instance, this methodology could be applied to a cohort undergoing open surgical techniques for MTLE, where objective assessments of resection cavities can be made (see below). It could also have utility in providing information that can help with manipulating the variables that can achieve effective thermal ablation of brain tumors and cerebral metastases in difficult-to-access locations ${ }^{80,81}$ and previously irradiated brain tumors. $^{82,83}$ In malignant brain tumor models, such as GBM where extent of tumor ablation may have prognostic significance, quantitative MR volumetric analysis via this methodology can provide more accurate information about survival to patient care teams, patients and their families ${ }^{80,84-86}$ - an application that may be extended to quantify the extent of tumor resection in craniotomy cases as well. Quantitative MR volumetric analysis can additionally provide information that 
can affect how physicians utilize stereotactic radiosurgery in treatment of brain tumors, ${ }^{87,88}$ arterio-venous malformations $^{89}$ and cavernous malformations ${ }^{90}$ by augmenting current understanding of treatment related MR changes.

Despite the aforementioned benefits, there are some obvious limitations. While the utilization of automated image analysis software offers a standardized, and therefore objective method for co-registration, outputs still required visual inspection to ensure verification and consistency. This is a subjective process in itself and provides an opportunity where observer variability can be introduced. In clinical practice, gross alignment of pre- and postoperative imaging may be sufficient for analyses; however, volumetric accuracy is likely to be better assessed with quantitative metrics that characterize image co-registration errors.

Secondly, in our workflow some of the image sets are manually segmented, which is a time-consuming and laborious process. Yet, we felt that it was necessary in order to accurately define the ablation cavity. Additionally, challenges to manual segmentation are already indicated above. Semi-automated segmentation methods can help in some cases, but whether such methods are time-efficient is arguable. As indicated above, through this modular approach, fully automated segmentation protocols may be eventually able to completely obviate the need for manual segmentation, but until then any automated segmentation method will need to be supervised and verified visually.

Thirdly, Freesurfer automated segmentation as it functions currently in this algorithm, is computationally intensive and can take as many as eleven hours to complete. While this is a significant improvement from whole brain manual segmentation, which can take an expert tracer approximately a week, one can foresee the challenge in clinical applications and in automated segmentation of very large data sets.

\section{Considerations with Open Cranial Surgery}

In preliminary work, we have begun to test this methodology in patients that have undergone ATL for MTLE (See Figure 4). Our experience with our ATL cohort brought to light a few important

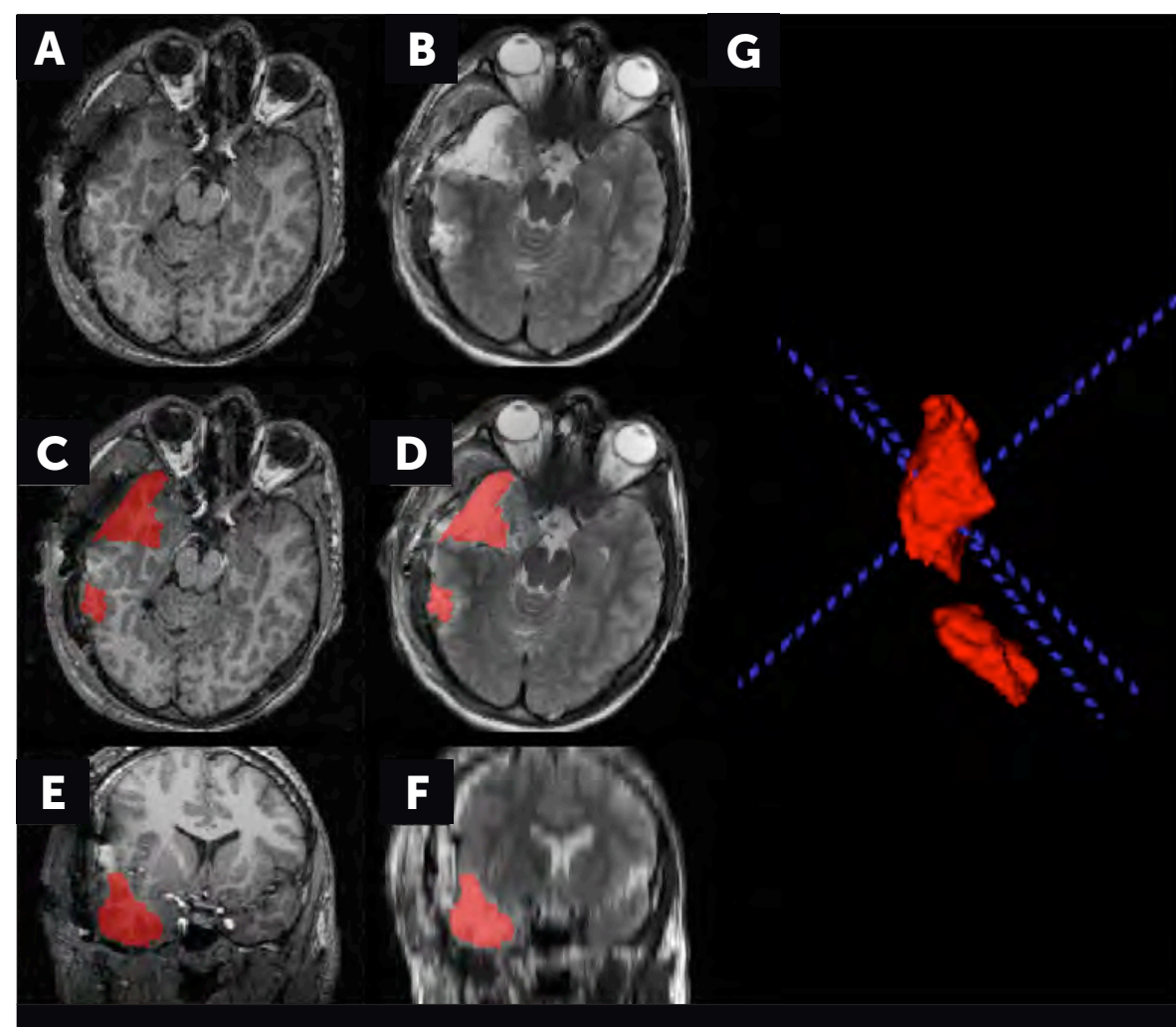

Figure 4

A, B Axial view of post-implant and post-resection cavitites; C, D Axial view of post-implant and post-resection cavitites; E,F Coronal view of segmented post-implant and post-resection cavitites; G 3D volumteric reconstruction of resection cavity; saggital views not shown.

considerations. Firstly, unlike in stereotactic laser procedures where postoperative MR images are obtained immediately after the case, in open surgical procedures this is not routinely done. In fact, it may be potentially precarious to the patient.

Postoperative MR images are typically obtained several hours to days after the case. Defining the optimal time to obtain the postoperative image is essential since brain morphological changes attributable to factors such as brain edema, and re-expansion occur more frequently in the immediate postoperative setting. Additionally, MR signal intensities of postoperative debris including fluid, air, blood products and contused brain within and around the resection bed evolve over time and may occasionally obscure the real boundaries of the resection cavity.

Typically, non-rigid registration depends on image-processing based methods such as mutual information based similarity ${ }^{91}$, entropy-based alignment ${ }^{92}$, and block matching ${ }^{93}$, but without considering the mechanical properties of the structural and anatomical features borne in the images. Image-based methods yield an anatomical model that provides a static geometrical representation that needs to be complemented with a biomechanical model ${ }^{94,95}$ adding a level of complexity. Non-linear finite element strategies can then be used to implement patient-specific models that are used to predict brain shift deformation. ${ }^{94-96}$ Devising many of these patient-specific brain deformation models can have high computation times and can therefore be costly. ${ }^{94}$

Speed needs to be weighed with robustness and accuracy in creating a suitable brain-shift deformation model that is personalized, effective, and yet sufficiently generalizable. This poses a significant challenge in image registration for open craniotomies. However, once resolved, this methodology will provide an objective means of comparing parameters 
of resection. For instance, volumes of resection cavities amongst MTLE subjects obtained can be used to assess varying resection techniques presently being employed (i.e. ATL, SAH, Lesionectomy) for MTLE more objectively.

\section{CONCLUSIONS}

In this retrospective study, we sought to describe a methodology that can be used to evaluate variables such as optimal laser placement and ablation volumes, which may be determinants of seizure outcomes in stereotactic laser amygdalohippocampectomy.

The presented methodology is modular in approach, which allows virtually any image analysis software package equipped with image visualization, pre-processing, image registration and segmentation toolkits to be employed, making the components of this workflow algorithm subject to constant review and allowing for constant optimization.

\section{ACKNOWLEDGEMENTS}

No financial support was required in the preparation of this manuscript.

\section{REFERENCES}

1. M. R. Sperling et al., "Temporal lobectomy for refractory epilepsy," JAMA 276(April 1986), 470-475 (1996) [doi:10.1001/ jama.1996.03540060046034].

2. S. Wiebe et al., "A Randomized, Controlled Trial of Surgery for Temporal-Lobe Epilepsy," N. Engl. J. Med. 345(5), 311-318 (2001) [doi:10.1056/NEJM200108023450501].

3. J. T. Willie et al., "Real-time magnetic resonance-guided stereotactic laser amygdalohippocampotomy for mesial temporal lobe epilepsy," Neurosurgery 74(6), 569-584 (2014) [doi:10.1227/NEU.0000000000000343].

4. M. Kalina et al., "Stereotactic amygdalohippocampectomy for temporal lobe epilepsy: promising results in 16 patients," Epileptic Disord. 9 Suppl 1, S68-S74 (2007) [doi:10.1684/epd.2008.0158]

5. R. Liscak et al., "Stereotactic radiofrequency amygdalohippocampectomy in the treatment of mesial temporal lobe epilepsy," Acta Neurochir. (Wien). 152(8), 1291-1298 (2010) [doi:10.1007/s00701-010-0637-2].
6. H. Malikova et al., "Microsurgical and stereotactic radiofrequency amygdalohippocampectomy for the treatment of mesial temporal lobe epilepsy: different volume reduction, similar clinical seizure control," Stereotact. Funct. Neurosurg. 88(1), 42-50 (2010) [doi:10.1159/000268741].

7. H. Malikova et al., "Stereotactic radiofrequency amygdalohippocampectomy: two years of good neuropsychological outcomes," Epilepsy Res. 106(3), 423-432 (2013) [doi:10.1016/j.eplepsyres.2013.07.009].

8. Z. Wu et al., "Efficacy and safety of a new robot-assisted stereotactic system for radiofrequency thermocoagulation in patients with temporal lobe epilepsy," Exp. Ther. Med. 7(6), 1728-1732 (2014) [doi:10.3892/ etm.2014.1620].

9. Z. Vojtěch et al., "Long-term seizure outcome after stereotactic amygdalohippocampectomy," Acta Neurochir. (Wien). 156(8), 1529-1537 (2014) [doi:10.1007/s00701-0142126-5].

10. C. Wu et al., "191 Methodology for critical evaluation of laser placement in stereotactic laser ablation for mesial temporal lobe epilepsy: A pilot study" (2014)

11. R. Gross et al., "Stereotactic laser amygdalohippocampotomy for mesial temporal lobe epilepsy: Collective experience from 7 singlecenter, prospective, investigator-initiated studies" (2014).

12. I. A. Awad et al., "Extent of resection in temporal lobectomy for epilepsy. I. Interobserver analysis and correlation with seizure outcome," Epilepsia 30(6), 756-762 (1989).

13. M. H. Nayel, I. A. Awad, and H. Luders, "Extent of mesiobasal resection determines outcome after temporal lobectomy for intractable complex partial seizures," Neurosurgery 29(1), 55-60; discussion 60-61 (1991).

14. A. R. Wyler, B. P. Hermann, and G. Somes, "Extent of medial temporal resection on outcome from anterior temporal lobectomy: a randomized prospective study," Neurosurgery 37(5), 982-990; discussion 990-991 (1995).

15. A. M. Kanner et al., "Tailored anterior temporal lobectomy. Relation between extent of resection of mesial structures and postsurgical seizure outcome," Arch. Neurol. 52(2), 173-178 (1995).

16. L. Bonilha et al., "Value of extent of hippocampal resection in the surgical treatment of temporal lobe epilepsy," Arq. Neuropsiquiatr. 62(August 2003), 15-20 (2004) [doi:10.1590/ S0004-282X2004000100003].

17. L. Bonilha et al., "Does resection of the medial temporal lobe improve the outcome of temporal lobe epilepsy surgery?," Epilepsia 48(3), 571-578 (2007) [doi:10.1111/j.15281167.2006.00958.x].

18. M. Jones-Gotman et al., "Learning and retention of words and designs following excision from medial or lateral temporal-lobe structures," Neuropsychologia 35(7), 963-973 (1997).
19. J. Schramm, "Temporal lobe epilepsy surgery and the quest for optimal extent of resection: A review," Epilepsia 49(8), 1296-1307 (2008) [doi:10.1111/j.1528-1167.2008.01604.x].

20. T. Tanriverdi et al., "Long-term seizure outcome after mesial temporal lobe epilepsy surgery: corticalamygdalohippocampectomy versus selective amygdalohippocampectomy," J. Neurosurg. 108(3), 517-524 (2008) [doi:10.3171/JNS/2008/108/3/0517].

21. J. Schramm et al., "Randomized controlled trial of $2.5-\mathrm{cm}$ versus $3.5-\mathrm{cm}$ mesial temporal resection in temporal lobe epilepsy - Part 1: Intent-to-treat analysis," Acta Neurochir. (Wien). 153(2), 209-219 (2011) [doi:10.1007/ s00701-010-0900-6].

22. C. Wu et al., "The Effects of Anatomic Variations on Stereotactic Laser Amygdalohippocampectomy and a Proposed Protocol for Trajectory Planning," Neurosurgery 00, 1 (2015) [doi:10.1227] NEU.0000000000000767].

23. A. M. Siegel et al., "Relationships between MR-imaged total amount of tissue removed, resection scores of specific mediobasal limbic subcompartments and clinical outcome following selective amygdalohippocampectomy," Epilepsy Res. 6, 56-65 (1990) [doi:10.1016/0920-1211(90)90009-K].

24. A. M. Dale, B. Fischl, and M. I. Sereno, "Cortical surface-based analysis. I. Segmentation and surface reconstruction," Neuroimage 9(2), 179-194 (1999) [doi:10.1006/nimg.1998.0395].

25. A. M. Dale and M. I. Sereno, "Improved Localizadon of Cortical Activity by Combining EEG and MEG with MRI Cortical Surface Reconstruction: A Linear Approach," J. Cogn. Neurosci. 5(2), 162-176 (1993) [doi:10.1162/ jocn.1993.5.2.162]

26. B. Fischl and A. M. Dale, "Measuring the thickness of the human cerebral cortex from magnetic resonance images," Proc. Natl. Acad. Sci. U. S. A. 97(20), 11050-11055 (2000) [doi:10.1073/pnas.200033797].

27. B. Fischl, A. Liu, and A. M. Dale, "Automated manifold surgery: constructing geometrically accurate and topologically correct models of the human cerebral cortex," IEEE Trans. Med. Imaging 20(1), 70-80 (2001) [doi:10.1109/42.906426]

28. B. Fischl et al., "Whole brain segmentation automated labeling of neuroanatomical structures in the human brain," Neuron 33(3), 341-355 (2002)

29. B. Fischl et al., "Automatically parcellating the human cerebral cortex," Cereb. Cortex 14(1), 11-22 (2004).

30. B. Fischl et al., "Sequence-independent segmentation of magnetic resonance images," Neuroimage 23 Suppl 1 S69-S84 (2004) [doi:10.1016/j.neuroimage.2004.07.016] 
31. B. Fischl, M. I. Sereno, and A. M. Dale, "Cortical surface-based analysis. II: Inflation, flattening, and a surface-based coordinate system," Neuroimage 9(2), 195-207 (1999) [doi:10.1006/nimg.1998.0396].

32. B. Fischl et al., "High-resolution intersubject averaging and a coordinate system for the cortical surface," Hum. Brain Mapp. 8(4). 272-284 (1999).

33. X. Han et al., "Reliability of MRI-derived measurements of human cerebral cortical thickness: the effects of field strength, scanner upgrade and manufacturer," Neuroimage 32(1), 180-194 (2006) [doi:10.1016/j.neuroimage.2006.02.051].

34. J. Jovicich et al., "Reliability in multi-site structural MRI studies: effects of gradient non-linearity correction on phantom and human data," Neuroimage 30(2), 436-443 (2006) [doi:10.1016/j.neuroimage.2005.09.046].

35. F. Ségonne et al., "A hybrid approach to the skull stripping problem in MRI," Neuroimage 22(3), 1060-1075 (2004) [doi:10.1016/j.neuroimage.2004.03.032].

36. M. Reuter, H. D. Rosas, and B. Fischl, "Highly accurate inverse consistent registration: a robust approach," Neuroimage 53(4), 1181-1196 (2010) [doi:10.1016/j.neuroimage.2010.07.020].

37. B. Fischl et al., "Whole brain segmentation: Automated labeling of neuroanatomical structures in the human brain," Neuron 33, 341-355 (2002) [doi:10.1016/S08966273(02)00569-X].

38. A. R. Khan, L. Wang, and M. F. Beg, "FreeSurfer-initiated fully-automated subcortical brain segmentation in MRI using Large Deformation Diffeomorphic Metric Mapping," Neuroimage 41, 735-746 (2008) [doi:10.1016/j.neuroimage.2008.03.024].

39. A. Fedorov et al., "3D Slicer as an image computing platform for the Quantitative Imaging Network," Magn. Reson. Imaging 30(9), 1323-1341 (2012) [doi:10.1016/j. mri.2012.05.001]

40. P. A. Yushkevich et al., "User-guided 3D active contour segmentation of anatomical structures: Significantly improved efficiency and reliability," Neuroimage 31(3). 1116-1128 (2006) [doi:10.1016/j.neuroimage.2006.01.015].

41. W. Schroeder, K. Martin, and W. Lorensen "The design and implementation of an object-oriented toolkit for 3D graphics and visualization," Proc. 7th Conf. Vis. IEEE Comput. Soc. Press (1996).

42. R. A. Morey et al., "A comparison of automated segmentation and manual tracing for quantifying hippocampal and amygdala volumes," Neuroimage 45(3), 855-866, Elsevier B.V. (2009) [doi:10.1016/j.neuroimage.2008.12.033].
43. H. R. Pardoe et al., "Hippocampal volume assessment in temporal lobe epilepsy: How good is automated segmentation?," Epilepsia 50(12), 2586-2592 (2009) [doi:10.1111/j.15281167.2009.02243.x].

44. J. Dewey et al., "Reliability and validity of MRI-based automated volumetry software relative to auto-assisted manual measurement of subcortical structures in HIV-infected patients from a multisite study," Neuroimage 51(4), 1334-1344, Elsevier Inc. (2010) [doi:10.1016/j.neuroimage.2010.03.033]

45. T. M. Doring et al., "Evaluation of hippocampal volume based on MR imaging in patients with bipolar affective disorder applying manual and automatic segmentation techniques," J. Magn. Reson. Imaging 33(3), 565-572 (2011) [doi:10.1002/jmri.22473].

46. W. S. Tae et al., "Validation of hippocampal volumes measured using a manual method and two automated methods (FreeSurfer and IBASPM) in chronic major depressive disorder," Neuroradiology 50(7), 569-581 (2008) [doi:10.1007/s00234-008-0383-9].

47. A. Akhondi-Asl et al., "Hippocampal volumetry for lateralization of temporal lobe epilepsy: Automated versus manual methods," Neuroimage 54, S218-S226, Elsevier Inc. (2011) [doi:10.1016/j.neuroimage.2010.03.066]

48. H. Kim et al., "Automatic hippocampal segmentation in temporal lobe epilepsy: Impact of developmental abnormalities," Neuroimage 59(4), 3178-3186, Elsevier Inc. (2012) [doi:10.1016/j.neuroimage.2011.11.040]

49. M.-P. Hosseini et al., "Statistical validation of automatic methods for hippocampus segmentation in MR images of epileptic patients.," Conf. Proc. Annu. Int. Conf. IEEE Eng. Med. Biol. Soc. IEEE Eng. Med. Biol. Soc. Annu. Conf. 2014, 4707-4710 (2014) [doi:10.1109/EMBC.2014.6944675].

50. C. R. Jack et al., "Anterior temporal lobes and hippocampal formations: normative volumetric measurements from MR images in young adults," Radiology 172(2), 549-554 (1989) [doi:10.1148/radiology.172.2.2748838]

51. R. S. Briellmann et al., "Seizure-associated hippocampal volume loss: a longitudinal magnetic resonance study of temporal lobe epilepsy," Ann. Neurol. 51(5), 641-644 (2002) [doi:10.1002/ana.10171].

52. F. Cendes, "Progressive hippocampal and extrahippocampal atrophy in drug resistant epilepsy," Curr. Opin. Neurol. 18(2), 173-177 (2005).

53. P. Demaerel and A. L. Baert, Recent Advances in Diagnostic Neuroradiology, Springer (2013).

54. F. M. Gunning-Dixon et al., "Aging of cerebral white matter: a review of MRI findings," Int. J. Geriatr. Psychiatry 24(2), 109-117 (2009) [doi:10.1002/gps.2087].

55. N. Raz and K. M. Rodrigue, “Differential aging of the brain: patterns, cognitive correlates and modifiers," Neurosci. Biobehav. Rev. 30(6), 730-748 (2006) [doi:10.1016/j. neubiorev.2006.07.001]
56. Y. Ge et al., "Age-related total gray matter and white matter changes in normal adult brain Part II: quantitative magnetization transfer ratio histogram analysis," AJNR. Am. J. Neuroradiol. 23(8), 1334-1341 (2002).

57. A. M. Fjell et al., "Critical ages in the life course of the adult brain: Nonlinear subcortical aging," Neurobiol. Aging 34(10), 2239-2247 (2013) [doi:10.1016/j.neurobiolaging.2013.04.006]

58. A. M. Hedman et al., "Human brain changes across the life span: a review of 56 longitudinal magnetic resonance imaging studies," Hum. Brain Mapp. 33(8), 1987-2002 (2012) [doi:10.1002/hbm.21334].

59. R. S. N. Liu et al., "Progressive neocortical damage in epilepsy," Ann. Neurol. 53(3), 312-324 (2003) [doi:10.1002/ana.10463].

60. R. S. N. Liu et al., "Cerebral damage in epilepsy: a population-based longitudinal quantitative MRI study," Epilepsia 46(9), 1482-1494 (2005) [doi:10.1111/j.15281167.2005.51603.x].

61. B. C. Bernhardt et al., "Longitudinal and crosssectional analysis of atrophy in pharmacoresistant temporal lobe epilepsy," Neurology 72(20), 1747-1754 (2009) [doi:10.1212/01. wnl.0000345969.57574.f5].

62. S. K. Warfield, K. H. Zou, and W. M. Wells, "Simultaneous truth and performance level estimation (STAPLE): an algorithm for the validation of image segmentation," IEEE Trans. Med. Imaging 23(7), 903-921 (2004) [doi:10.1109/TMI.2004.828354].

63. C. R. Jack et al., "MR imaging-based volume measurements of the hippocampal formation and anterior temporal lobe: validation studies," Radiology 176(1), 205-209 (1990) [doi:10.1148/radiology.176.1.2353093].

64. F. Klauschen et al., "Evaluation of automated brain MR image segmentation and volumetry methods," Hum. Brain Mapp. 30(4), 13101327 (2009) [doi:10.1002/hbm.20599].

65. G. Sánchez-Benavides et al., "Manual validation of FreeSurfer's automated hippocampal segmentation in normal aging, mild cognitive impairment, and Alzheimer Disease subjects," Psychiatry Res. 181(3), 219-225 (2010) [doi:10.1016/j.pscychresns.2009.10.011]

66. A. Butts, "Freesurfer Vs. Manual Tracing: Detecting Future Cognitive Decline In Healthy Older Adults At-Risk For Alzheimer's Disease," in Dissertations (2009 -) (2013).

67. L. P. Clarke et al., "The Quantitative Imaging Network: NCl's Historical Perspective and Planned Goals," Transl. Oncol. 7(1), 1-4 (2014)

68. K. K. Leung et al., "Automated cross-sectional and longitudinal hippocampal volume measurement in mild cognitive impairment and Alzheimer's disease," Neuroimage 51(4), 1345-1359 (2010) [doi:10.1016/j.neuroimage.2010.03.018]. 
69. O. Colliot et al. "Discrimination between Alzheimer disease, mild cognitive impairment, and normal aging by using automated segmentation of the hippocampus," Radiology 248(1), 194-201 (2008) [doi:10.1148/radiol.2481070876].

70. J. H. Morra et al., "Validation of a fully automated 3D hippocampal segmentation method using subjects with Alzheimer's disease mild cognitive impairment, and elderly controls," Neuroimage 43(1), 59-68 (2008) [doi:10.1016/j.neuroimage.2008.07.003].

71. R. B. Zipursky et al., "Volumetric MRI assessment of temporal lobe structures in schizophrenia," Biol. Psychiatry 35, 501-516 (1994) [doi:10.1016/0006-3223(94)90097-3].

72. M. R. Kaus et al., "Automated segmentation of MR images of brain tumors," Radiology 218(2), 586-591 (2001) [doi:10.1148/ radiology.218.2.r01fe44586].

73. L. M. Fletcher-Heath et al., "Automatic segmentation of non-enhancing brain tumors in magnetic resonance images," Artif. Intell. Med. 21(1-3), 43-63 (2001)

74. G. P. Mazzara et al., "Brain tumor target volume determination for radiation treatment planning through automated MRI segmentation," Int. J. Radiat. Oncol. Biol. Phys. 59(1), 300-312 (2004) [doi:10.1016/j. ijrobp.2004.01.026]

75. R. E. Hogan et al., "Mesial temporal sclerosis and temporal lobe epilepsy: MR imaging deformation-based segmentation of the hippocampus in five patients," Radiology 216(1), 291-297 (2000) [doi:10.1148/ radiology.216.1.r00jl41291]

76. L. Bonilha et al., "Voxel-based morphometry reveals gray matter network atrophy in refractory medial temporal lobe epilepsy," Arch. Neurol. 61(9), 1379-1384 (2004) [doi:10.1001/ archneur.61.9.1379]

77. M. Chupin et al., "Fully automatic segmentation of the hippocampus and the amygdala from MRI using hybrid prior knowledge," Med. Image Comput. Comput. Assist. Interv. 10(Pt 1), 875-882 (2007)

78. S. S. Keller et al., "Voxel based morphometry of grey matter abnormalities in patients with medically intractable temporal lobe epilepsy: effects of side of seizure onset and epilepsy duration," J. Neurol. Neurosurg. Psychiatry 73(6), 648-655 (2002) [doi:10.1136/ jnnp.73.6.648].

79. A. Hammers et al., "Automatic detection and quantification of hippocampal atrophy on MRI in temporal lobe epilepsy: a proof-of-principle study," Neuroimage 36(1), 38-47 (2007) [doi:10.1016/j.neuroimage.2007.02.031].

80. A. M. Mohammadi et al., "The role of laser interstitial thermal therapy in enhancing progression-free survival of difficult-toaccess high-grade gliomas: a multicenter study," Cancer Med. 3(4), 971-979 (2014) [doi:10.1002/cam4.266].
81. J. L. Schroeder et al., "Laser interstitial thermal therapy as a novel treatment modality for brain tumors in the thalamus and basal ganglia," Photonics Lasers Med. 3(2) (2014) [doi:10.1515/plm-2013-0053]

82. T. R. Patel and V. L. S. Chiang, "Laser interstitial thermal therapy for treatment of postradiosurgery tumor recurrence and radiation necrosis," Photonics Lasers Med. 3(2), 95-105 (2014) [doi:10.1515/plm-2013-0057].

83. M. S. Rao et al., "Magnetic resonanceguided laser ablation improves local control for postradiosurgery recurrence and/or radiation necrosis.," Neurosurgery $74(6)$. 658-667; discussion 667 (2014) [doi:10.1227/ NEU.0000000000000332]

84. A. H. Hawasli et al., "Magnetic resonance imaging-guided focused laser interstitial thermal therapy for intracranial lesions: Single-institution series," Neurosurgery 73(6), 1007-1017 (2013) [doi:10.1227/ NEU.0000000000000144].

85. S. Missios et al., "Prognostic factors of overall survival after laser interstitial thermal therapy in patients with glioblastoma," Photonics Lasers Med. 3(2) (2014) [doi:10.1515/ plm-2013-0051].

86. S. Missios, K. Bekelis, and G. H. Barnett, "Renaissance of laser interstitial thermal ablation," Neurosurg. Focus 38(3), E13 (2015) [doi:10.3171/2014.12.FOCUS14762].

87. J. S. Kuo et al., "Gamma Knife Radiosurgery for Benign Cavernous Sinus Tumors: Quantitative Analysis of Treatment Outcomes," Neurosurgery 54(6), 1385-1394 (2004) [doi:10.1227/01. NEU.0000124750.13721.94]

88. A. Varma et al., "Gamma knife radiosurgery for glomus jugulare tumors: volumetric analysis in 17 patients," Neurosurgery 59(5), 10301036; discussion 1036 (2006) [doi:10.1227/01. NEU.0000245596.46581.B2].

89. O. Cohen-Inbar et al., "A quantitative analysis of adverse radiation effects following Gamma Knife radiosurgery for arteriovenous malformations," J. Neurosurg., 1-9 (2015) [doi:10.3171/2014.10.JNS142264].

90. J. M. Frischer et al., "Microsurgery and radiosurgery for brainstem cavernomas: effective and complementary treatment options," World Neurosurg. 81(3-4), 520-528 (2014) [doi:10.1016/j.wneu.2014.01.004].

91. P. Viola and I. Wells, W.M., "Alignment by maximization of mutual information," Proc. IEEE Int. Conf. Comput. Vis. 24(2), 137-154 (1997) [doi:10.1109/ICCV.1995.466930].

92. S. K. Warfield et al., "Brain shift computation using a fully nonlinear biomechanical model," Med. Image Comput. Comput. Assist. Interv. 8(Pt 2), 583-590 (2001).

93. J. Dengler and M. Schmidt, "The Dynamic Pyramid-A Model For Motion Analysis with Controlled Continuity," Int. J. Pattern Recognit. Artif. Intell. 02(02), 275-286, World Scientific Publishing Company (1988) [doi:10.1142/S0218001488000170].
94. J. Ma et al. "On the Effects of Model Complexity in Computing Brain Deformation for Image-Guided Neurosurgery," in Computational Biomechanics for Medicine: Soft Tissues and the Musculoskeletal System, A. Wittek, P. M. F. Nielsen, and K. Miller, Eds., pp. 51-61, Springer Science \& Business Media (2011).

95. H. 'E Delingette and N. Ayache, "Building Patient-Specific Physical and Physiological Computational Models from Medical Images," in Handbook of Biomedical Imaging, N. Paragios, J. Duncan, and N. Ayache, Eds., pp. 169-182, Springer US, Boston, MA (2015) [doi:10.1007/978-0-387-09749-7]

96. A. Wittek, T. Hawkins, and K. Miller, "On the unimportance of constitutive models in computing brain deformation for image-guided surgery," Biomech. Model. Mechanobiol. 8(1), 77-84 (2009) [doi:10.1007/ s10237-008-0118-1].

97. D. W. Roberts et al., "Intraoperative brain shift and deformation: a quantitative analysis of cortical displacement in 28 cases," Neurosurgery 43(4), 749-758; discussion 758-760 (1998) 